\title{
LA INCLUSIÓN COMO EJE TRANSVERSAL EN EL DESARROLLO DE COMPETENCIAS DE LOS INGENIEROS DE SISTEMAS
}

\section{THE INCLUSION AS A TRANSVERSAL AXIS IN THE DEVELOPMENT OF COMPETENCES OF SYSTEM ENGINEERS}

\author{
Bertha Alice Naranjo Sánchez ${ }^{1}$ \\ David Fernando Zorrilla Ortiz ${ }^{2}$ \\ Universidad Politécnica Salesiana
}

\section{RESUMEN}

Los estudiantes de la Carrera de Ingeniería de Sistemas en su proceso de aprendizaje deben

1 Bertha Alice Naranjo Sánchez, bnaranjo@ups. edu.ec, Universidad Politécnica Salesiana, 0996473514, Guayaquil, Ecuador, https://orcid.org/0000-0002-4386-2335 2 David Fernando Zorrilla Ortiz, dzorrilla@est.ups. edu.ec, Universidad Politécnica Salesiana, 0986176250, Guayaquil, Ecuador, https://orcid.org/0000-0002-7349-908X desarrollar competencias que le permitan ejercer actividades en el campo profesional. Un eje transversal de toda Carrera es la inclusión de personas con discapacidad. Con la Ley de Discapacidades y el Código de Trabajo vigentes en el Ecuador que exigen la inclusión laboral de personas con discapacidad, los profesionales en Sistemas tienen la responsabilidad de hacer efectiva la inclusión y brindar todas las herramientas necesarias para facilitar la 
autonomía laboral en igualdad de derechos en los diferentes puestos de trabajo. El presente artículo describe cómo los estudiantes de la Carrera de Ingeniería de Sistemas perciben el fortalecimiento de sus competencias profesionales hacia la inclusión dentro de su proceso de enseñanza-aprendizaje.

\section{PALABRAS CLAVE}

Inclusión, Competencias, Discapacidad, TIC, inserción laboral.

\section{ABSTRACT}

Students of the Systems Engineering Career in their learning process must develop skills that allow them to exercise activities in the professional field. A transversal axis of every career is the inclusion of people with disabilities. With the Disabilities Law and the Labor Code in force in Ecuador that require the labor inclusion of people with disabilities, systems professionals have the responsibility of making inclusion effective and providing all the necessary tools to facilitate work autonomy in equal rights in the different Jobs. This article describes how the students of the Systems Engineering Career perceive the strengthening of their professional competences towards inclusion in their teachinglearning process.

\section{KEYWORDS}

Inclusion, Professional skills, Disability, ICT, labor insertion.

\section{INTRODUCCIÓN}

En el Ecuador, el Código de Trabajo vigente obliga a las empresas a cumplir hasta con un $4 \%$ de inclusión de personas con discapacidad en la nómina de personal de cada una de las organizaciones, teniendo para ello la obligación de proveer todos los recursos necesarios que permitan que el entorno, espacio o puesto de trabajo estén adecuados a la persona con discapacidad.

Muchas de las actividades que permiten este proceso inclusivo las debe desarrollar el profesional de sistemas quien tiene la responsabilidad de proveer las TIC (Tecnologías de Información y Comunicación) y todas las herramientas de apoyo que le permitirán a las personas con discapacidad (PCD) desarrollar todas o casi todas sus actividades con autonomía.

Así en el proceso formativo las Universidades deben preparar a los profesionales con las competencias y herramientas necesarias a través de los ejes transversales, uno de ellos es la inclusión. Dentro de la academia los estudiantes deben enfrentar diversas situaciones reales que podrían presentársele en el futuro permitiéndoles resolver desde el campo técnico las necesidades laborales que le darán esa experiencia que hará de su organización una empresa inclusiva.

Este artículo permite conocer cómo se desarrollan las competencias transversales en una entidad de educación superior en la Carrera de Ingeniería en Sistemas y cómo los estudiantes perciben este proceso de aprendizaje inclusivo.

\section{MARCO TEÓRICO}

El total de personas con discapacidad en el Ecuador a Diciembre de 2019 es de 479.910, de este valor en la ciudad de Guayaquil existen 78.733 personas con discapacidad, esto es un $16,40 \%$ de dicha población, de los cuales el $47,73 \%$ poseen discapacidad física, 22,21 discapacidad intelectual, 12,68\% auditiva, 10,52\%discapacidad visual y un $6,86 \%$ discapacidad psicosocial, distribuidos en porcentajes acorde al grado de discapacidad, tal como se aprecia en la figura1. (CONADIS, 2019) 


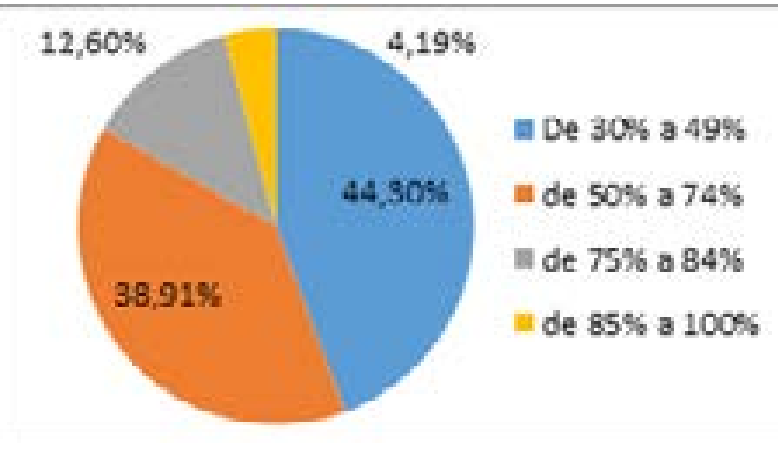

Figura 1.- Porcentaje de personas con discapacidad en la ciudad de Guayaquil, según el Grado de discapacidad.

Fuente: Ministerio de Salud Pública, Diciembre 2019.

\section{Elaborado por autores}

Tal como se observa en la figura 1 , el $83,21 \%$ de la población posee discapacidades comprendidas entre los rangos 30 a $49 \%$ y 50 a $74 \%$, mientras que el $16,79 \%$ poseen grados de discapacidad más severa. (CONADIS, 2019)

En la figura 2 se observa el grupo etario más numeroso que integra el $58,14 \%$ de personas comprendidas entre 25 y 64 años, siendo el género masculino aquel que cuenta con un $58,40 \%, 0,01 \%$ LGBTI y el femenino con un $41,59 \%$. Todas ellas con posibilidad de ser insertadas en el mercado laboral (CONADIS, 2019).

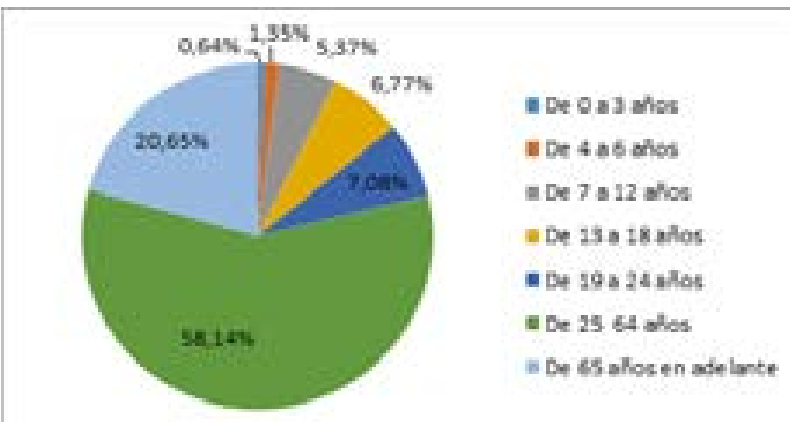

Figura 2.- Porcentaje de personas con discapacidad en la ciudad de Guayaquil, según el grupo etario.

Fuente: Ministerio de Salud Pública, Diciembre 2019.

Elaborado por: Autores
Así, 45.775 personas se encuentran en edad productiva y demandan apoyos o ayudas tecnológicas para poder desarrollar su labor en igualdad de condiciones acorde al marco normativo vigente, sólo en la ciudad de Guayaquil.

De acuerdo al Código de trabajo, Art 42 numeral 33, en el Ecuador, "El empleador público o privado, que cuente con un número mínimo de veinticinco trabajadores, está obligado a contratar, al menos, a una persona con discapacidad, en labores permanentes que se consideren apropiadas en relación con sus conocimientos, condición física y aptitudes individuales, observándose los principios de equidad de género y diversidad de discapacidad, en el primer año de vigencia de esta Ley. En el segundo año, la contratación será del $1 \%$ del total de los trabajadores, en el tercer año el $2 \%$, en el cuarto año el 3\% hasta llegar al quinto año en donde la contratación será del $4 \%$ del total de los trabajadores, siendo ese el porcentaje fijo que se aplicará en los sucesivos años. Esta obligación se hace extensiva a las empresas legalmente autorizadas para la tercerización de servicios o intermediación laboral" (República del Ecuador, 2014). Este porcentaje permite hacer efectivo el justo derecho de inclusión laboral que demandan las personas con discapacidad.

Para que las empresas puedan hacer la inclusión efectiva, el Código de trabajo, Art 42 numeral 35 precisa "Las empresas e instituciones, públicas o privadas, para facilitar la inclusión de las personas con discapacidad al empleo, harán las adaptaciones a los puestos de trabajo de conformidad con las disposiciones de la Ley de Discapacidades, normas INEN sobre accesibilidad al medio físico y los convenios, acuerdos, declaraciones internacionales legalmente suscritos por el país" (República del Ecuador, 2014), su incumplimiento genera multa para el empleador acorde a la última actualización del código en el 2014. 
Para poder hacer efectiva la inclusión laboral descrita en elArtículo33y 35 citadoanteriormente, es necesario que el personal del área de TIC (Tecnología de información y Comunicación) de las empresas puedan brindarle todo el apoyo a la persona con discapacidad que ha sido contratada, por ello a través del análisis de los requerimientos de hardware y software que demanda el puesto de trabajo y acorde a sus necesidades particulares, el personal del departamento de TI (Tecnología de Información) hará las adecuaciones o ajustes pertinentes, identificando, seleccionando y adaptando en el caso de ser necesario las tecnologías de apoyo - TIC adaptativas que permitan brindarle al nuevo colaborador la autonomía suficiente para el desarrollo de sus labores.

Conscientes de la necesidad del cumplimiento de la ley de discapacidad, su reglamento y el Código de trabajo vigente, con el objetivo de hacer efectiva la inclusión, la Universidad Politécnica Salesiana (UPS), a través del grupo de investigación denominado Tecnología de información y comunicación asociados a discapacidad (TICAD) y su Carrera de Ingeniería de Sistemas desarrollaron un proyecto denominado Inclusión educativa de estudiantes con discapacidad (INCLED) citado en (Naranjo Sánchez, Naranjo Sánchez, Mora Saltos, \& Huilcapi Subia, 2015), el cual permitió que los docentes de la Carrera introduzcan en los contenidos curriculares el eje transversal de la inclusión para fortalecer las competencias de los futuros profesionales.

Si bien la UPS es una universidad inclusiva que ha venido trabajando en el tema de inclusión y discapacidad desde sus inicios (Tomalá Cabezas \& Naranjo, 2019), se consideró necesario plantear alternativas de solución en este sentido dentro del contenido curricular de la Carrera. Como indica (Area, 2010) "Las universidades deben formar a las personas en las competencias necesarias de modo que puedan afrontar los desafíos derivados de las nuevas relaciones sociales, y responder a las nuevas necesidades tecnológicas, económicas, científicas y culturales".

Así en la sede Guayaquil desde el 2013 se han establecido procesos que permiten que los estudiantes de la Carrera de Sistemas trabajen en este tema teniendo en cuenta a la inclusión como eje transversal, en ese contexto el desarrollo de las competencias juegan un importante papel.

Una competencia se entiende como un proceso complejo mediante el cual la persona realiza actividades y resuelve problemas mediante la articulación del saber, el saber hacer y el saber ser, con autonomía, crítica y creatividad. Una competencia involucra simultáneamente conocimientos, desempeños y actitudes. Los conocimientos se relacionan con la dimensión del saber; los desempeños con el hacer, involucran procedimientos, desarrollo de productos y estrategias; y las actitudes están ubicadas en la dimensión del ser junto a la motivación, la iniciativa, la disposición y otras características que pueden identificarse en la personalidad de quien tiene la competencia (Boude Figueredo \& Medina Rivilla, 2011). Existen diversos tipos de competencias: genéricas, específicas, personales, sociales, profesionales, transversales, todas ellas relevantes para la formación de un profesional.

Una definición de competencia genérica es la que establece el proyecto Tuning y Tuning LA que dice: "Una competencia genérica será toda competencia de tipo instrumental, interpersonal y sistémica comunes a cualquier profesión, tales como la capacidad de aprender, de tomar decisiones, de solucionar problemas, de diseñar proyecto, etc., y una competencia específica será toda competencia relacionada con el área de estudio o profesión, cruciales para cualquier título y referidas a la especificidad propia de un 
campo de estudio" (Boude Figueredo \& Medina Rivilla, 2011).

Las competencias personales, sociales y profesionales son necesarias para poder afrontar los continuos cambios que se imponen en todos los campos y actividades, así como los rápidos avances que presenta la sociedad en torno al uso generalizado de las TIC en todas las actividades humanas y ámbitos sociales que afectan la práctica educativa. Por tal razón, se espera que las TIC ayuden a desarrollar nuevas formas de aprendizaje, comunicación y modos de interacción con las fuentes del saber y el conocimiento como vehículos que fomenten la circulación, el uso, el acceso, la representación y la creación de información (Hernandez, Gamboa, \& Ayala, 2014).

Las competencias transversales son un conjunto de habilidades de amplio alcance que afectan a distintas clases de tareas y que se desarrollan en situaciones distintas, por lo que son ampliamente generalizables y transferibles, dando como resultado una ejecución profesional eficaz. (Gisbert Cervera, Cela-Ranilla, \& Isus Barado, 2011).

Así, una persona demuestra que es competente a través de su desempeño, cuando es capaz de resolver con éxito diferentes situaciones de forma flexible y creativa. (Hernandez, Gamboa, \& Ayala, 2014).

(Ruay, Jara, \& López, 2013), señalan que se pretende que la formación de profesionales tenga en cuenta la articulación del ser y el hacer, no de un aprendizaje teórico o de desarrollo de habilidades descontextualizadas, como tradicionalmente se ha dado (Arboleda J. C., 2016), (Arboleda J. C., 2008A).

Por ello la Carrera de Ingeniería en Sistemas fortalece las competencias de los estudiantes haciéndoles enfrentar retos, problemas y casos, en los que combinan la teoría con la aplicación práctica, desarrollo de proyectos y resolución de problemas basados en tics inclusivas o tecnologías de apoyo para ponerlas a disposición de las personas con discapacidad al tiempo que van desarrollando su ser "en una comprensión edificadora” (Arboleda J. C., 2016), (Arboleda J. C., 2008A).

\section{METODOLOGÍA}

Para el desarrollo de este trabajo se aplica el método descriptivo, basado en entrevistas abiertas $y$ un cuestionario que permiten evidenciar de los actores del proceso, docentes y estudiantes de la Carrera de Ingeniería de Sistemas, el trabajo realizado en la temática inclusión y discapacidad. El cuestionario dirigido a estudiantes contó con preguntas en escalas dicotómicas y de Lickert así como preguntas de selección. La población estuvo integrada por un total de 200 estudiantes que corresponden a 5 cursos evaluados, con un rango de edades comprendido entre 21 a 28 años, los cuales respondieron el cuestionario en línea para conocer si se están desarrollando las competencias profesionales con el eje transversal de la inclusión, en la Carrera y si se han tratado temas que favorezcan la inclusión laboral de personas con discapacidad.

\section{DESARROLLO}

Las actividades que la Carrera desarrolla teniendo como eje transversal a la inclusión y discapacidad se encuentran circunscritas a la academia, investigación, extensiones sociales y asociacionismo.

El enfoque inclusivo se desarrolla en las asignaturas de la academia, en procesos de investigación así como en proyectos de vinculación con la sociedad y fueron ejecutados en instituciones de PCD, entre otras organizaciones sociales.

Las asignaturas en los que se trataron los contenidos curriculares y temáticas inclusivas 
corresponden a: Control de Calidad, Arquitectura de computadoras, Auditoría Informática, Sistemas Operativos I, Sistemas Operativos II, Proyectos de Software, Programación, Sistemas de Información, Investigación, Ingeniería de Requerimientos, entre otras.

Los temas que los estudiantes han revisado en este proceso para fomentar sus competencias inclusivas fueron glosario de inclusión, ley de discapacidad y el reglamento respectivo, marco normativo, normas técnicas INEN e ISO, normas de accesibilidad, diseño universal, accesibilidad web, entre otros.

Como resultado del cuestionario aplicado se obtuvo que el $100 \%$ de estudiantes indicó estar trabajando como eje transversal la inclusión y discapacidad en las asignaturas seleccionadas, que en su vida académica han interactuado con personas con discapacidad a través de los proyectos desarrollados, que en los proyectos de aula se ha trabajado el eje transversal de la inclusión de personas con discapacidad, lo cual fue corroborado en las entrevistas realizadas a los docentes y descritos en la información de malla curricular proporcionada por la Carrera.

Los proyectos académicos que realizaron los estudiantes de las 5 asignaturas seleccionadas para este trabajo investigativo tienen un componente inclusivo, lo cual fue confirmado tanto por los docentes como por los delegados de instituciones de la red RAITI consultados.

A través de los proyectos realizados en las asignaturas, los estudiantes en un $85 \%$ indicaron haber aprendido a hacer adaptaciones del puesto de trabajo para las personas con discapacidad, basándose en TIC.

\section{CONCLUSIONES}

Este proceso desarrollado en la UPS en la Carrera de Ingeniería de Sistemas, viene aplicándose desde el 2013 y no ha sido un proceso sencillo, según los docentes entrevistados, pero en la actualidad se encuentra aplicándose con más presencia en más asignaturas, así la Carrera puede ofrecer a la sociedad un profesional más competente en diversos campos y en especial con conocimiento en tecnologías de apoyo y TIC asistidas que favorecerán la inserción laboral de personas con discapacidad.

El fortalecimiento de las competencias personales, genéricas, sociales y transversales, aportan significatividad de los contenidos acorde a lo indicado por los estudiantes. Esto va alineado a la descripción general de la Carrera que la UPS ofrece, que dice "Formar Ingenieros e Ingenieras en Computación con una sólida base de conocimiento técnico y científico sustentada en un enfoque humanista y de responsabilidad ética y social, capaces de liderar innovar y desarrollar respuestas tecnológicas que permitan contribuir a la búsqueda de soluciones para problemas de la sociedad y el desarrollo del país, alineado a estándares de calidad nacionales e internacionales" (Universidad Politecnica Salesiana, s.f.).

En este artículo se describe cómo la Carrera de Ingeniería de Sistemas de la UPS Sede Guayaquil ha logrado integrar dentro de su currículo estos conceptos que permitan que los futuros profesionales puedan contribuir con la inclusión laboral de personas con discapacidad, para ello en las diferentes asignaturas se los enfrenta a escenarios, proyectos y situaciones que permiten alcanzar ese objetivo. Los estudiantes adquieren conocimientos y práctica con el trabajo en escenarios reales que permiten fortalecer las competencias descritas en el perfil profesional de la carrera así como favorecer la inclusión de personas con discapacidad. 
Esta propuesta puede ser aplicada en otras Carreras y se podría trabajar en diversos ámbitos profesionales, también podría ser implementada en instituciones salesianas que mantienen la aplicación del asociacionismo salesiano como esquema optativo similar al voluntariado para estudiantes que deseen trabajar de forma directa, activa y participativa con la sociedad y en especial con las personas con discapacidad.

Se recomienda a otras Carreras y Universidades a integrar en la academia este $u$ otros ejes transversales tanto en el macro, meso y microcurrículo acorde a una planificación y/o actualización de malla curricular.

\section{AGRADECIMIENTOS}

Al grupo de investigación TICAD por el acceso a información del proyecto Inclusión Educativa de estudiantes con discapacidad en la UPS Sede Guayaquil.

A la Universidad Politécnica Salesiana, Carrera de Sistemas/Computación y a las instituciones que conforman la red RAITI (Red Académica de Apoyo e Investigación en Tecnologías inclusivas) por su participación como informantes de este proceso de investigación.

\section{REFERENCIAS BIBLIOGRÁFICAS}

Arboleda, J. C. (2008A). Aprendizaje y comprensión: del enfoque de competencias al enfoque de comprensiones y proyectos de vida. Rev. Eduación y Cultura(ํ․ 79).

Arboleda, J. C. (2016). Formación para la vida: de las competencias a la comprensión edificadora. Vol.4( $\left.\mathrm{N}^{\circ} .12\right), 20-25$.

Area, M. (2010). ¿Por qué formar en competencias informacionales y digitales en la educación superior? Universidad y Sociedad del Conocimiento, 1-4. Recuperado el 04 de 08 de 2019, de http://www.uoc.edu/ojs/index.php/rusc/ article/view/v7n2-area/v7n2-area

Boude Figueredo, O., \& Medina Rivilla, A. (2011). Desarrollo de competencias a través de un ambiente de aprendizaje mediado por TIC en educación superior. Educación Médica Superior(25(3)), 301311.

CONADIS. (2 de agosto de 2019). Resumenestadísticodediscapacidad. Recuperado el 23 de enero de 2020, de https://www. consejodiscapacidades.gob.ec/wpcontent/uploads/downloads/2019/08/ Resumen-estad\%C3\%ADstico-dediscapacidad.pdf

Gisbert Cervera, M., Cela-Ranilla, J. M., \& Isus Barado, S. (2011). Las simulaciones en entornos tic como herramienta para la formación en competencias transversales de los estudiantes universitarios. Teoría de la Educación. Educación y Cultura en la Sociedad de la Información, Vol. 11( $\mathrm{N}^{\circ}$. 1). Obtenido de https://www.redalyc.org/ pdf/2010/201014897015.pdf

Hernandez, C., Gamboa, A., \& Ayala, E. (2014). Competencias TIC para los docentes de Educación Superior. Congreso Iberoamericano de Ciencia, Tecnología, Innovación y Educación.

Naranjo Sánchez, B. A., Naranjo Sánchez, R. A., Mora Saltos, N., \& Huilcapi Subia, D. (2015). La Investigación y las TIC para la inclusión educativa de personas con discapacidad: Red RAITI. Educación para la paz, la comprensión $y$ el desarrollo de competencias. Universidad Politécnica Salesiana, Guayaquil, Ecuador.

República del Ecuador. (12 de Septiembre de 2014). https://www. consejodiscapacidades.gob.ec/wp- 
content/uploads/downloads/2015/04/ codigodeltrabajo.pdf. Recuperado el 05 de Agosto de 2019

Ruay, R., Jara, \& López. (2013). Desde una evaluación de contenidos a una evaluación de competencias. Colombia.

Tomalá Cabezas, L. A., \& Naranjo, B. A. (2019). Sistema de seguimiento académico a estudiantes. 6(1)(124-37).

Universidad Politecnica Salesiana. (s.f.). www. ups.edu.ec. Obtenido de https://www. ups.edu.ec/computacion-guayaquil 